\title{
Mental Health Care Consumption and Outcomes: Considering Preventative Strategies Across Race and Class
}

\author{
Barak Richman, Dan Grossman, Craig Chepke, \& Frank Sloan \\ Duke University Law School \\ Duke University Center for Health Policy \\ Duke University Medical Center
}

\begin{abstract}
In previous work (Richman 2007), we found that even under conditions of equal insurance coverage and access to mental healthcare providers, whites and high-income individuals consume more outpatient mental health services than nonwhites and low-income individuals. We follow-up that study to determine (1) whether nonwhite and low-income individuals obtain medical substitutes to mental healthcare, and (2) whether disparate consumption leads to disparate health outcomes. We find that nonwhites and low-income individuals are more likely than their white and high-income counterparts to obtain mental health care from general practitioners over mental healthcare providers, and nearly twice as likely not to follow up with a mental health provider after hospitalization with a mental health diagnosis. We further are unable to find any evidence that this leads to adverse health outcomes. These findings echo concern expressed in Richman (2007) that low-income and nonwhite individuals might be paying for health services that primarily benefit their white and more affluent coworkers.
\end{abstract}




\section{$\underline{\text { Introduction }}$}

Mandating mental health insurance is a popular legislative exercise. Despite intense partisan vitriol and a divided government, Congress passed—and President George W. Bush signed—-the Paul Wellstone and Pete Domenici Mental Health Parity and Addiction Equity Act of 2008. This 2008 Act substantially expanded Congress' previous mental health insurance mandate, the Mental Health Parity Act of 1996, and most states—dominated by both Democratic Republican administrations-have instituted their own mental health parity mandates. The most recent, and most expansive, chapter of requiring mental health insurance comes in the contested Patient Protection and Affordable Care Act (PPACA). One of PPACA's lesser known, and less controversial, provisions creates a mandated insurance benefit for certain mental health services (to be determined by rulemaking currently underway) for qualified group and individual plans. Unconsidered in debates over parity and mandated insurance is the financial impact of expanded mental health benefits on individual workers. If increases in insurance coverage - and the subsequent increases in the cost of insurance-are fully shifted to reductions in take-home pay, ${ }^{1}$ then it is important to consider whether insureds are receiving corresponding benefits.

Richman (2007) found that even under conditions of equal insurance coverage and access to mental healthcare providers, whites and high-income individuals consume more outpatient mental health services than nonwhites and low-income individuals. Since consumption of insured healthcare services amounts to an extraction of financial benefits, disparate consumption of healthcare translates into disparate collection of insurance dollars. Richman (2007) found that when controlling for income and other 
demographic variables, whites on average received four times as many mental health insurance dollars as African Americans and five times as many as Asians; and when controlling for race, the $75^{\text {th }}$ percentile wage earner received $65 \%$ more mental health services than the $25^{\text {th }}$ percentile wage earner. ${ }^{2}$ If insurance premiums withhold equal amounts of take-home pay for insured workers of all incomes (and it's only been presumed, but not proven, that it is ${ }^{3}$ ), then insurance mental health insurance benefits facilitate a regressive wealth transfer from low- to high-income workers and from nonwhite to white insureds.

Richman (2007) puts a dollar figure on what most observers surely suspected: that whites and high-income individuals take greater advantage of, and thus extract more financial gain from, a given menu of insurance benefits. Prior research confirms that high-income insureds are less deterred by copayments and other cost-sharing burdens than lower-income individuals with the same insurance benefits (Keeler, et al., 1988; Newhouse et al., 1993). Affluent individuals also are better at navigating through medical bureaucracies to obtain desired providers, high-quality treatment, and medical advocacy (Bloche 2001), and there remains significant evidence that African Americans receive inferior care and attention in the US health system (Institute of Medicine, 2003). Moreover, consumption disparities in mental health services are further explained by different attitudes towards mental healthcare. Nonwhites have been shown to attribute a larger stigma to mental illnesses and seeking mental health care than whites (U.S. Surgeon General, 2001), and there is evidence that nonwhites are more likely than whites to use social support systems and religious participation as alternatives to seeking care from mental healthcare providers (Williams \& Neighbors, 2006). 
Nonetheless, despite the consequent wealth transfer, mandating coverage for mental healthcare might still be a desirable policy. If it is determined that receiving outpatient mental health care prevents costly mental health hospitalizations, or if receiving services from an outpatient mental health provider is shown to have greater benefits (at lower costs) than receiving services from alternative sources, then perhaps coverage is desirable and low-users of mental health care should be encouraged to consume more. In this paper, we examine whether low-users of insurance-covered mental health benefits—specifically, low-income and nonwhite individuals—obtain medical substitutes to mental healthcare, and we examine whether those substitutes lead to worse health outcomes than mental health services. We also test, more generally, whether disparate consumption of outpatient mental health services leads to disparate mental health outcomes. We find that nonwhites and low-income individuals are more likely than their white and high-income counterparts to obtain mental health care from general practitioners over mental healthcare providers. We further are unable to find any evidence that this leads to adverse health outcomes.

These findings echo concern expressed in Richman (2007) that low-income and nonwhite individuals might be paying for health services that primarily benefit their white and more affluent coworkers. They also sound caution to the agencies currently writing regulations to implement PPACA. If the benefits packages required under PPACA cover services that reflect the healthcare consumption behaviors of the elite, then the Act could engineer a regressive wealth transfer that injures the individuals the Act is designed to help most. 


\section{Description of the Data}

Our data is obtained from a major university health system in North Carolina (UHS). UHS provides health insurance to more than 20,000 employees in over six counties in central North Carolina. ${ }^{4}$ We obtained limited access to deidentified records of each employee’s health claims from 2001 through 2004, yielding almost 92,000 personyear observations. Each health claim includes information on the services provided, the associated diagnosis, and the amounts paid by both the insurer and patient. The data also reveal each individual's race, job category (from which education and income are derived $^{5}$ ), and insurance benefits.

The demographic profile of the population remains stable for the period under study. Approximately 68\% of sample is white and $24 \%$ is African American, the median annual income rises gradually from about $\$ 36,000$ to $\$ 40,500$ over the four years, and the $75^{\text {th }}$ and $25^{\text {th }}$ percentile incomes range from approximately $\$ 47,800$ to $\$ 51,000$ and $\$ 28,600$ to $\$ 30,500$, respectively. These figures are roughly reflective of the demographic profile of both Durham County (in which UHS is located) and North Carolina. $^{6}$

UHS offers its employees a menu of insurance coverage options for different employee-paid premiums, including an HMO (selected by over 70\% of employees), a more expensive PPO with a wider network of participating providers (selected by about $15 \%$ of employees), and other managed care options, some of which were terminated and replaced during the period of study. The different plans offer slightly varying copayments, deductibles, and rates of coinsurance for most medical services, and they also present different copayments for going to out-of-network providers. However, most 
of these insurance plans offer the same package of mental health benefits, including identical copayments, network, and coverage of services, so there is far less variation across plans for these benefits. In 2004, for example, three of the four insurance plans, subscribed collectively by $87 \%$ of the employees, offered identical mental health and substance abuse benefits, with the remaining 13\% with a BCBS plan enjoying almost identical financial coverage but for a wider network. ${ }^{7}$

The dataset offers an unusual opportunity to examine healthcare consumption when access is held constant across race and income. Most data sources on healthcare consumption, such as the Medical Expenditure Panel Survey (MEPS), rely on selfreported surveys of populations in which individuals have different insurance benefits and confront assorted barriers to care. In contrast, all of the individuals in the UHS dataset have comprehensive health insurance with nearly uniform mental health coverage. Moreover, the Raleigh-Durham metropolitan area is home to many providers (including two academic medical centers), so individuals in the data live near a hospital and a physician practice, and since the data includes UHS employees, a great number of individuals work at or right next to healthcare institutions. Thus, the UHS population faces very few logistical and institutional barriers to care, and observed consumption disparities can be attributed to other factors. ${ }^{8}$

\section{Methods \& Results}

To examine both the effect and efficacy of insurance coverage for outpatient mental health services, we first examine whether low-income and non-white individuals 
seek substitutes to mental health services. We then examine whether those substitutes, or forgoing mental health care altogether, lead to adverse health outcomes.

Consumption Patterns. We first sought to determine whether race or income is systematically associated with variation in mental healthcare seeking behavior. Our claims data reveals at least three ways insureds can use insurance benefits to obtain outpatient mental health care: receiving care from a mental healthcare professional, filling prescriptions for psychotropic pharmaceuticals, or visiting a general practitioner. The claims data determined whether an insured sought care from a mental health provider or a general practitioner. We separated pharmaceutical claims for psychotropics from other prescriptions based on their NDC codes, and we used International Classification of Diseases $9^{\text {th }}$ edition (ICD-9) diagnoses codes - relying only on the primary codes - to determine whether an insured's visit to a general practitioner included treatment for a mental illnesses.

Insureds were separated into four mutually exclusive categories: (1) individuals who sought care from an outpatient mental healthcare provider (including those who also obtained psychotropic pharmaceuticals and/or sought care from a general practitioner and received a mental illnesses diagnosis), (2) individuals who filled a prescription for psychotropics (including those who sought care from a general practitioner and received a mental illnesses diagnosis) but did not obtain care from an outpatient mental healthcare provider, (3) individuals who sought care from a general practitioner and received a mental illnesses diagnosis but neither obtained care from a mental healthcare provider nor filled a prescription for psychotropics, and (4) individuals who received no form of 
mental healthcare. We labeled these categories Outpatient Mental Health (OMH), Psychotropics/No-OMH, GP-Only, and No Care.

We employed a multinomial logit test to compare how race and income affected an individual's probability of being in one of the three consumption categories. Table 1 shows the relative risk ratios (RRRs) that capture the comparative probabilities. The 0.29 RRR for African Americans in the OMH category is the probability an African American will consume outpatient mental healthcare divided by the probability he/she will not consume any care. Since whites are the reference group, it means African Americans are only $29 \%$ ( $p<0.001)$ as likely as whites to be in the OMH group compared to the No Care group. Asians are even less likely than whites to be in the OMH group compared to the No care group, and income is found to increase the relative probability of consuming mental healthcare. These findings, with their significant magnitudes, corroborate those in Richman (2007).

One question raised in Richman (2007) is whether nonwhites and low-income workers obtained mental healthcare through alternative sources. Table 1 indicates that African Americans and Asians are also much less likely to obtain mental healthcare from mental health providers and through psychotropic prescriptions than whites, but are more likely to see a general practitioner for a mental health problem (RRR: 1.24; $p<0.001)$ than not seek treatment at all, compared to whites. Income, however, appears to have an opposite effect on these alternative sources, and lower incomes are associated with greater likelihoods of receiving care from general practitioners and psychotropic prescriptions compared to not seeking help, while higher incomes are associated with greater likelihoods of receiving care from mental health professionals compared to not 
seeking help. So, while nonwhites are less likely than whites to consume mental health care from mental health providers or through prescription medicines, low-income individuals appear to substitute GPs and prescriptions for mental health providers.

Table 2 further explores different consumption patterns by executing a multinomial logit only for those who seek some kind of care for a mental illness and excludes the No Care group. These findings confirm that rising incomes are associated with declining use of general practitioners for mental health care and increasing use of mental healthcare providers. Also, both Asians (RRR: 2.84; $\mathrm{p}<0.001$ ) and African Americans (RRR: 2.91; $\mathrm{p}<0.001$ ) are nearly 3 times as likely to seek care for mental illnesses from GPs than through psychotropic prescriptions compared to whites, while African Americans are just two-thirds as likely (RRR: 0.65; $\mathrm{p}<0.001$ ) to seek care from mental health providers than through psychotropic prescriptions compared to whites.

These results illustrate that both the race and income variables independently (i.e. when each one is controlled for the other) are associated with different patterns of healthcare consumption. As a general matter, we see significant differences in how individuals of different races and with different incomes seek healthcare for mental illnesses, as low-income and nonwhite individuals are more inclined compared to whites to obtain care from GPs than mental health professionals. We also observe that nonwhites are less likely than whites to seek outpatient mental healthcare or prescription medications, whereas low-income individuals, compared to their more affluent coworkers, appear to substitute care from GPs and prescriptions for psychotropics for outpatient mental healthcare. 


\section{Incidence of Mental Illness and Effectiveness of Mental Healthcare. Differences}

in consumption patterns are difficult to interpret meaningfully without evaluating the effectiveness of the alternative forms of care. We begin by examining whether outpatient mental healthcare, compared to GP visits and psychotropics (which are covered in standard care, not by a mental healthcare benefit) reduce the likelihood of an adverse outcome. We use hospitalizations associated with mental illnesses as an indicator of an adverse outcome, which we gathered from three sources. We identified any individual hospitalized with a primary diagnosis of mental disorder (ICD-9 codes 290-319), any insured who sought treatment at an emergency room and received a primary diagnosis of mental disorder, and any patient who received mental health care with a service code that denoted inpatient treatment (which largely included hospital patients who had an inpatient mental health consult). With these three sources, we identified 297 individuals who were hospitalized at least once.

Since mental illnesses prevent many individuals from maintaining their employment, we employed a competing risk model to compare the probability of hospitalization with the likelihoods that individuals will leave our sample of insured, which occurs when an employee leaves the UHS workplace. The competing risk model permits a comparison of two alternative risks for identical groups while controlling for differences in the sizes of the groups of interest. The results in Table 3 reveal that the probabilities of African Americans, Asians, and whites being hospitalized for a mental illness are statistically indistinguishable, while low-income employees are more likely to be hospitalized than their higher-income co-workers (Hazard ratio (HR): 0.82; $\mathrm{p}=0.001$ ) (high- 
income employees are also more likely to leave the employment sample, probably because of better outside labor market opportunities).

Table 3 therefore dispels, in part, one potential explanation for the results in Tables 1 and 2, that differences in consumption across race reflect differences in need. Table 3 instead suggests that nonwhites are about as likely to require hospitalization as whites, and thus their lower levels of consumption cannot be solely attributed to differences in the incidence of mental illness. The combined results in Tables 1-3 also conform to research relying on survey data revealing that ethnic and racial minorities experience lower prevalence rates of acute mental illnesses than whites but are equally likely (and often more likely) to present severe major disorders and debilitating mental illnesses (Williams et al. 2007).

The bigger question begged by Tables 3 is whether interventions by medical professionals can reduce the probability of a hospitalization associated with a mental illness, and whether some interventions are more effective than others (Tables 1-3 also suggest that low-income individuals are more likely to be hospitalized yet are less likely to seek outpatient mental health care, which additionally invite further testing of the efficacy of interventions). Table 4 introduces interventions into the competing risk model and offers little evidence that the interventions work. Individuals who consume outpatient mental healthcare are more than nine times as likely to be hospitalized as individuals who receive no care, and individuals who fill prescriptions for psychotropics are more than three times as likely.

Of course, Table 4's results are readily explained by the endogeneity of the consumption patterns, since individuals who seek mental healthcare of any sort are 
revealing some mental illness, and individuals with some form of illness are more likely to be hospitalized. The problem of controlling for underlying condition is an enormous empirical challenge throughout all these efforts to evaluate the effectiveness of alternative interventions. In Table 5, we add to the competing risk model our own severity index, in which a psychiatrist assigned a 1-10 value for each mental illness-related ICD9 diagnosis, with 10 being the most severe (see Appendix A for more information on the severity index). Each individual who received a diagnosis from either a general practitioner or an outpatient mental healthcare provider thus received a severity score, and in order to allow the severity index to predict hospitalizations, we based the severity score on the diagnosis individuals received before they were hospitalized (if they were hospitalized at all). The problem with employing this metric, aside from its reliance on approximations, is that it assigns a zero to all individuals who do not receive any diagnosis. Thus, since more than one-quarter of those hospitalized did not visit a GP or mental health provider before being hospitalized, and thus did not receive a diagnosis, the metric is necessarily biased downward. Nonetheless, in Table 5 the severity index is positively associated with the likelihood of hospitalization, and including it in the model makes the medical interventions less associated with hospitalizations (suggesting that the severity measure does help solve the endogeneity problem). More important, even when controlling for severity, the results do not indicate that receiving care from outpatient mental health providers reduces the likelihood of hospitalization. Receiving care from a general practitioner - a service covered by standard insurance benefits, not by mental health benefits - does appear to reduce the probability of hospitalization. 
For a robustness check, and to pursue another path to control for the severity of the underlying medical condition, we examined only the 297 individuals who were hospitalized for a mental illness. Even though these individuals were hospitalized under different conditions and for different illnesses, their severity is much more homogeneous than that of the whole sample. Moreover, each hospitalized individual is, at time of discharge, given an appointment to see an outpatient mental healthcare provider within the first few weeks of discharge, with regular visits scheduled thereafter. We therefore can test to see if these post-hospitalization instructions are followed, and we can test to see if race or income affects the probability an individual will miss, or refuse to attend, those followup appointments.

To test for "failure" to attend post-hospitalization outpatient appointments, we determine whether within the first four months of discharge there is a 90-day period in which a formerly hospitalized patient does not visit an outpatient mental health provider. We employ a competing risk model that compares the probability of an adherence failure across race and income, with results shown in Table 6. Here again, African Americans and Asians, controlling for income, exhibit a lower propensity to visit outpatient mental healthcare providers, even shortly after being discharged for a hospitalization (though the small sample size keeps the Asian coefficient from being statistically significant, with a pvalue of 0.23 ). Income does not affect follow-up behavior, suggesting that the refusals of nonwhites might be a function of cultural preferences rather than financial means.

In Table 7, we test whether the failure to follow post-discharge instructions has adverse consequences. We use rehospitalization as an adverse outcome, and we determine whether discharged individuals are hospitalized after 14 days (to ensure that the second 
admission reflects a second event, rather than a recurrence) but within one year of the date of initial discharge. We then employ a competing risk model to calculate whether the likelihood of rehospitalization is affected by race, income, or failure to pursue postdischarge outpatient mental healthcare. Table 7 reveals that there is little evidence that failure to follow-up increases the probability of a rehospitalization. In fact, a "failure" to follow-up with an outpatient mental health provider is closer to decreasing, rather than increasing, the likelihood of a rehospitalization (p-value is 0.26 ), although this also might be a problem of unobserved severity - individuals who fail to follow-up might have less severe illnesses. These analyses that focus on hospitalized insureds, however, have a much smaller sample size, and thus are less likely to produce significant results.

Table 8 reveals where discharged patients seek care, including those who fail to follow-up with mental healthcare providers. Here again, like the results in Tables 1 and 2, African Americans appear to prefer seeking care from general practitioners (or forgo care altogether) than visit mental healthcare providers. These results are even more striking than Tables 1 and 2 since they follow a severe event that was accompanied by instructions to see a mental healthcare provider, and like Table 1, African Americans are more likely that whites, post-hospitalization, to seek care from a GP. Table 9 offers similar results for the six months prior to an initial hospitalization. Of individuals who are hospitalized for mental illnesses, African Americans are more likely to seek care from GPs than from mental health providers. 


\section{Discussion \& Conclusion}

While policymakers who mandate insurance benefits might think that promoting insurance thereby promotes desirable health outcomes, our results offer little support. Nonwhites and low-income individuals do not take advantage of their mental health benefits at the same rates as their white and more affluent coworkers, and to the degree that they seek care for mental illnesses, they are more likely to seek care from general practitioners. Differences in consumption patterns across race are also evident among those who are hospitalized, both before and after hospitalization. The posthospitalization findings are especially striking since upon discharge, every patient is instructed to seek outpatient mental healthcare. The variation in adherence rates following hospitalization is a sharp illustration of the fragmentation of delivering mental healthcare, as insureds receive care from GPs, hospitalists, and mental health providers with little coordination.

Despite differences in consumption patterns, especially between African Americans and whites but also between Asians and whites and across income, we find no evidence that these differences affect the probability of hospitalizations for mental illnesses. Specifically, receiving care from a mental health provider does not reduce the probability of hospitalization, and following a hospitalization, receiving outpatient care from a mental health provider does not reduce the probability of rehospitalization.

In short, we find nothing to temper the provisional conclusions in Richman (2007). It appears that white and affluent workers take greater advantage of the mental health insurance benefit than their nonwhite and lower-income co-workers, that nonwhites, especially African Americans, are significantly more likely to seek care from 
general practitioners than from mental healthcare providers, and that there is no statistically significant evidence that receiving outpatient care from a mental healthcare provider reduces the likelihood of adverse mental health.

The limitations of these results should be recognized. The studied population works in a university setting, and it is unclear how generalizable the findings are. Moreover, relying on hospitalizations as a measure for adverse mental illnesses is fairly coarse, and more sensitive measurements - such as lost workdays or surveyed responses - would improve our ability to measure effectiveness.

More important, it is not clear what drives these results. The potential causes for the consumption disparities range from different attitudes towards necessary care, enmeshed in ethnic histories with healthcare providers or cultural attitudes towards mental illnesses, to different preferences and needs for care, to discriminatory referral practices and the effectiveness of care. Much more needs to be known about how individuals engage with their insurance benefits and healthcare providers and whether those benefits and providers meet the needs of the insureds. Many of these questions can be further explored with employer claims data, and we also hope to supplement these econometric investigations with surveys and focus groups that inquire into attitudes and practices that shape healthcare-seeking behavior. Given the complexity of the studied behavior, employing multiple methodologies and several data sources might be necessary before arriving at meaningful conclusions about mental health interventions and benefits policies.

Nonetheless, we continue to produce findings that raise serious questions about the efficacy and fairness of mandating mental health benefits, as Congress (like many 
state legislatures) has done again. Insurance coverage of this kind continues to appear as transfer payments from non-whites to whites, and from low-income to higher-income individuals. Before insurance expansions spread further, serious attention should be given to studying how insurance benefits and our fragmented healthcare system can improve mental health outcomes without charging vulnerable populations for services they do not want or need. 


\section{$\underline{\text { Tables }}$}

Table 1. Multinomial logit: Relative risk ratios (RRR) of receiving mental healthcare from alternative providers compared to receiving no mental healthcare

\begin{tabular}{|c|c|c|c|c|c|c|}
\hline & \multicolumn{2}{|c|}{$\begin{array}{l}\text { Outpatient Mental } \\
\text { Health }(\mathrm{OMH})\end{array}$} & \multicolumn{2}{|c|}{$\begin{array}{l}\text { Psychotrophics/ } \\
\text { No OMH }\end{array}$} & \multicolumn{2}{|c|}{$\begin{array}{l}\text { General } \\
\text { Practitioner only }\end{array}$} \\
\hline & RRR & $P$ value & RRR & $\mathrm{P}$ value & RRR & $P$ value \\
\hline Male & 0.56 & 0.000 & 0.46 & 0.000 & 1.05 & 0.328 \\
\hline Age & 1.02 & 0.000 & 1.05 & 0.000 & 1.03 & 0.000 \\
\hline African American & 0.29 & 0.000 & 0.43 & 0.000 & 1.24 & 0.000 \\
\hline Asian & 0.24 & 0.000 & 0.23 & 0.000 & 0.65 & 0.001 \\
\hline Income & 1.03 & 0.002 & 0.94 & 0.000 & 0.91 & 0.000 \\
\hline
\end{tabular}

Source: UHS Human Resources

$\mathrm{N}=31640$

Omitted reference group is "No Care"

RRR - Relative risk ratio

Covariates not shown include type of insurance, income missing, year of service

${ }^{*} p<0.05,{ }^{* *} p<0.01,{ }^{* \star *} p<0.001$

Table 2. Multinomial logit: Relative risk ratios (RRR) of receiving alternative forms of mental healthcare for those who obtain some form of mental health care

\begin{tabular}{lrrrr}
\hline & \multicolumn{3}{c}{ Outpatient Mental } & \multicolumn{2}{c}{$\begin{array}{l}\text { General } \\
\text { practitioner only }\end{array}$} \\
\cline { 2 - 5 } & Realth $(\mathrm{OMH})$ & \multicolumn{2}{c}{ practue } \\
\hline Male & 1.26 & 0.000 & 2.31 & 0.000 \\
Age & 0.97 & 0.000 & 0.98 & 0.000 \\
Black & 0.65 & 0.000 & 2.91 & 0.000 \\
Asian & 0.99 & 0.936 & 2.84 & 0.000 \\
Income & 1.10 & 0.000 & 0.96 & 0.065 \\
\hline
\end{tabular}

Source: UHS Human Resources

$\mathrm{N}=11129$

Omitted reference group is "Psychotrophics/no MH"

Covariates not shown include type of insurance, income missing, year of service ${ }^{*} \mathrm{p}<0.05,{ }^{* *} \mathrm{p}<0.01,{ }^{* * *} \mathrm{p}<0.001$ 
Table 3. Competing risk between the likelihood of hospitalization versus exiting the sample

\begin{tabular}{lrrrr}
\hline & \multicolumn{2}{c}{ Hospitalization } & \multicolumn{2}{c}{ Exiting sample } \\
\cline { 2 - 5 } & HR & P value & HR & P value \\
\hline Black & 0.80 & 0.148 & 1.12 & 0.477 \\
Asian & 0.71 & 0.286 & 1.63 & 0.137 \\
Income & 0.82 & 0.001 & 1.10 & 0.096 \\
\hline
\end{tabular}

Source: UHS Human Resources

$\mathrm{N}=31640$

Notes: HR - Hazard ratio

Covariates not shown include gender, type of insurance, income missing, age, year of service

${ }^{*} p<0.05,{ }^{* *} p<0.01,{ }^{* * *} p<0.001$

Table 4. Competing risk between the likelihood of hospitalization versus exiting the sample: Effect of mental health consumption

\begin{tabular}{lrrrr}
\hline & \multicolumn{2}{c}{ Hospitalization } & \multicolumn{2}{c}{ Exiting sample } \\
\cline { 2 - 5 } & HR & P value & HR & P value \\
\hline Black & 1.22 & 0.190 & 0.70 & 0.017 \\
Asian & 1.20 & 0.572 & 0.89 & 0.728 \\
Income & 0.80 & 0.000 & 1.12 & 0.071 \\
Outpatient mental health (OMH) & 9.01 & 0.000 & 0.08 & 0.000 \\
Psychotrophics/No OMH & 3.23 & 0.000 & 0.22 & 0.000 \\
General practitioner only & 1.60 & 0.101 & 0.43 & 0.003 \\
\hline
\end{tabular}

Source: UHS Human Resources

$\mathrm{N}=31640$

Notes: HR - Hazard ratio

Covariates not shown include gender, type of insurance, income missing, age, year of service

${ }^{*} p<0.05,{ }^{* *} p<0.01,{ }^{* \star *} p<0.001$ 
Table 5. Competing risk between the likelihood of hospitalization versus exiting the sample: Effect of mental health consumption and severity index

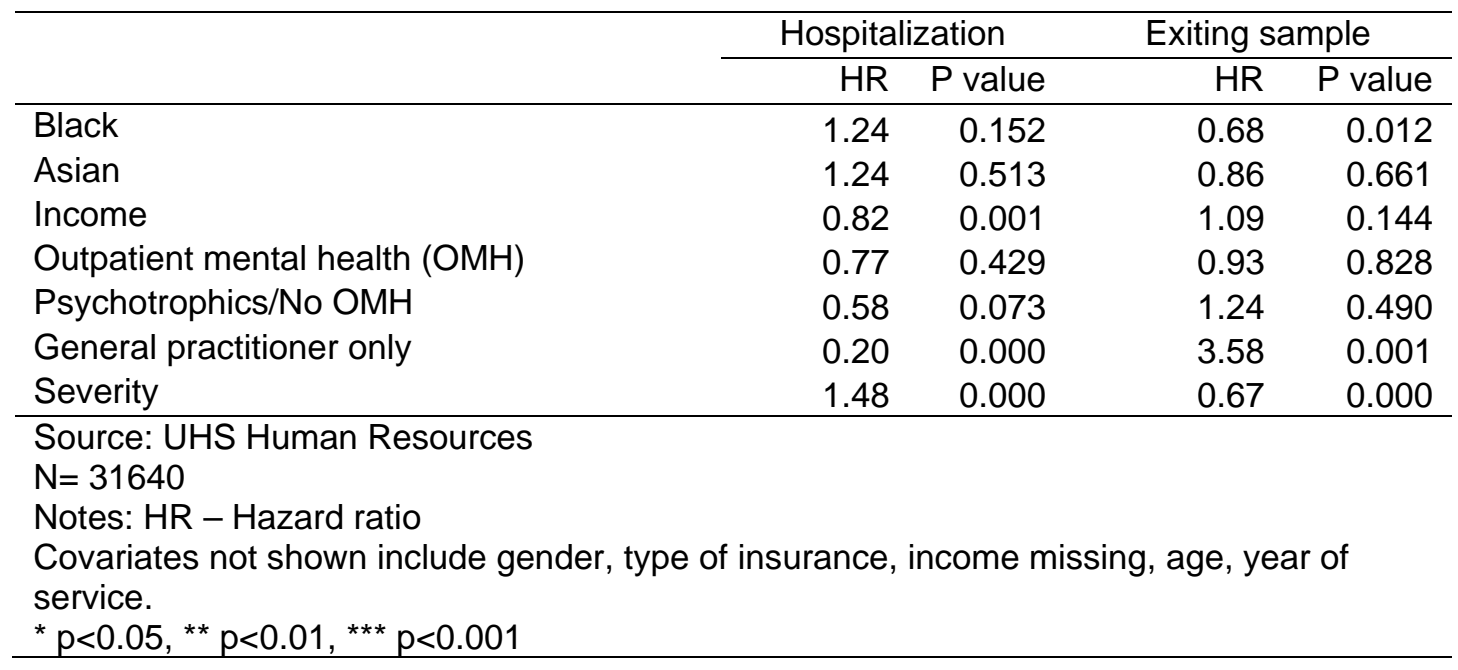


Table 6. Competing risk: Hazard ratios (HR) of failure to adhere to post-hospitalization follow-up treatment versus exiting the sample

\begin{tabular}{lrrrr}
\hline & \multicolumn{3}{c}{ Treatment failure } & \multicolumn{2}{c}{ Exiting sample } \\
\cline { 2 - 5 } & HR & P value & HR & P value \\
\hline Male & 1.21 & 0.265 & 1.23 & 0.653 \\
Age & 0.99 & 0.326 & 0.97 & 0.073 \\
Black & 1.92 & 0.000 & 0.52 & 0.201 \\
Asian & 1.64 & 0.231 & 2.75 & 0.287 \\
Income & 0.92 & 0.191 & 0.66 & 0.059 \\
Severity & 0.83 & 0.000 & 1.41 & 0.012 \\
\hline
\end{tabular}

Source: UHS Human Resources

$\mathrm{N}=297$

Notes: Treatment failure is not seeking mental health outpatient care for a period of longer than 90 days

Included covariates were year of service, type of insurance, and income missing. None were significant.

${ }^{*} p<0.05,{ }^{* *} p<0.01,{ }^{* \star *} p<0.001$

Table 7. Competing risk: Hazard ratios (HR) of rehospitalization within 1 year following initial hospitalization versus exiting the sample: Effect of Treatment failure

\begin{tabular}{lrlrl}
\hline & \multicolumn{3}{c}{ Rehospitalization } & Exiting sample \\
\cline { 2 - 5 } & HR & P value & HR & P value \\
\hline Male & 1.52 & 0.186 & 1.20 & 0.660 \\
Age & 1.02 & 0.186 & 0.96 & 0.036 \\
Black & 1.05 & 0.908 & 1.68 & 0.357 \\
Asian & 1.00 & 0.997 & 3.80 & 0.234 \\
Income & 0.92 & 0.480 & 1.00 & 0.982 \\
Severity & 1.12 & 0.277 & 0.97 & 0.809 \\
\hline Treatment failure & 0.65 & 0.263 & 0.36 & 0.070 \\
\hline
\end{tabular}

Source: UHS Human Resources

$\mathrm{N}=293$

Treatment failure is failure to see mental health provider for a period of 90 days within in the first four months following initial hospitalization.

Included covariates were year of service, type of insurance, and income missing. None were significant.

${ }^{*} p<0.05,{ }^{*} p<0.01,{ }^{* \star *} p<0.001$ 
Table 8. Multinomial logit: Relative risk ratio (RRR) of receiving mental healthcare from alternative providers in the 4 months after initial hospitalization versus exiting the sample

\begin{tabular}{lrrrrrr}
\hline & \multicolumn{3}{c}{$\begin{array}{c}\text { Outpatient Mental } \\
\text { Health }\end{array}$} & \multicolumn{2}{c}{$\begin{array}{c}\text { Psychotrophics or } \\
\text { general practioner } \\
\text { (No OMH) }\end{array}$} & \multicolumn{2}{c}{ Exit Sample } \\
\hline Male & RRR & P value & RRR & P value & RRR & P value \\
Age & 0.57 & 0.187 & 0.74 & 0.514 & 1.06 & 0.903 \\
Black & 1.02 & 0.318 & 1.01 & 0.518 & 1.00 & 0.953 \\
Asian & 0.27 & 0.004 & 0.57 & 0.249 & 0.24 & 0.008 \\
Income & 0.21 & 0.177 & 0.44 & 0.470 & 0.66 & 0.694 \\
Severity & 1.14 & 0.346 & 1.09 & 0.603 & 0.97 & 0.862 \\
Soun & 1.38 & 0.013 & 1.14 & 0.366 & 1.25 & 0.129
\end{tabular}

Source: UHS Human Resources

$\mathrm{N}=297$

Notes: Omitted reference group is "No Care"

${ }^{*} \mathrm{p}<0.05,{ }^{*} \mathrm{p}<0.01,{ }^{* \star *} \mathrm{p}<0.001$

Table 9. Multinomial logit: Relative risk ratios (RRR) of receiving mental healthcare from alternative providers in the 6 months prior to initial hospitalization

\begin{tabular}{lrrrrrr}
\hline & $\begin{array}{c}\text { Outpatient Mental } \\
\text { Health }\end{array}$ & $\begin{array}{c}\text { Psychotrophics/ No } \\
\text { OMH }\end{array}$ & \multicolumn{2}{c}{$\begin{array}{c}\text { General practitioner } \\
\text { only }\end{array}$} \\
\hline Male & RRR & P value & RRR & P value & RRR & P value \\
Age & 0.50 & 0.117 & 0.38 & 0.035 & 1.64 & 0.505 \\
Black & 1.04 & 0.087 & 1.04 & 0.087 & 1.03 & 0.439 \\
Asian & 0.08 & 0.000 & 0.19 & 0.000 & 4.07 & 0.162 \\
Income & 0.87 & 0.323 & 0.94 & 0.651 & 0.61 & 0.179 \\
Severity & 1.09 & 0.553 & 0.96 & 0.748 & 0.82 & 0.512 \\
Soun & 0.50 & 0.117 & 0.38 & 0.035 & 1.64 & 0.505
\end{tabular}

Source: UHS human resources

$\mathrm{N}=220$

Notes: Omitted reference group is "No Care"

Asians were excluded from this analysis due to insufficient sample size

${ }^{*} \mathrm{p}<0.05,{ }^{* *} \mathrm{p}<0.01,{ }^{* \star *} \mathrm{p}<0.001$ 


\section{Appendix A - The Severity Index}

The distribution of the severity of diagnoses in the sample appears to assume something akin to a normal distribution, without significant variation across race.

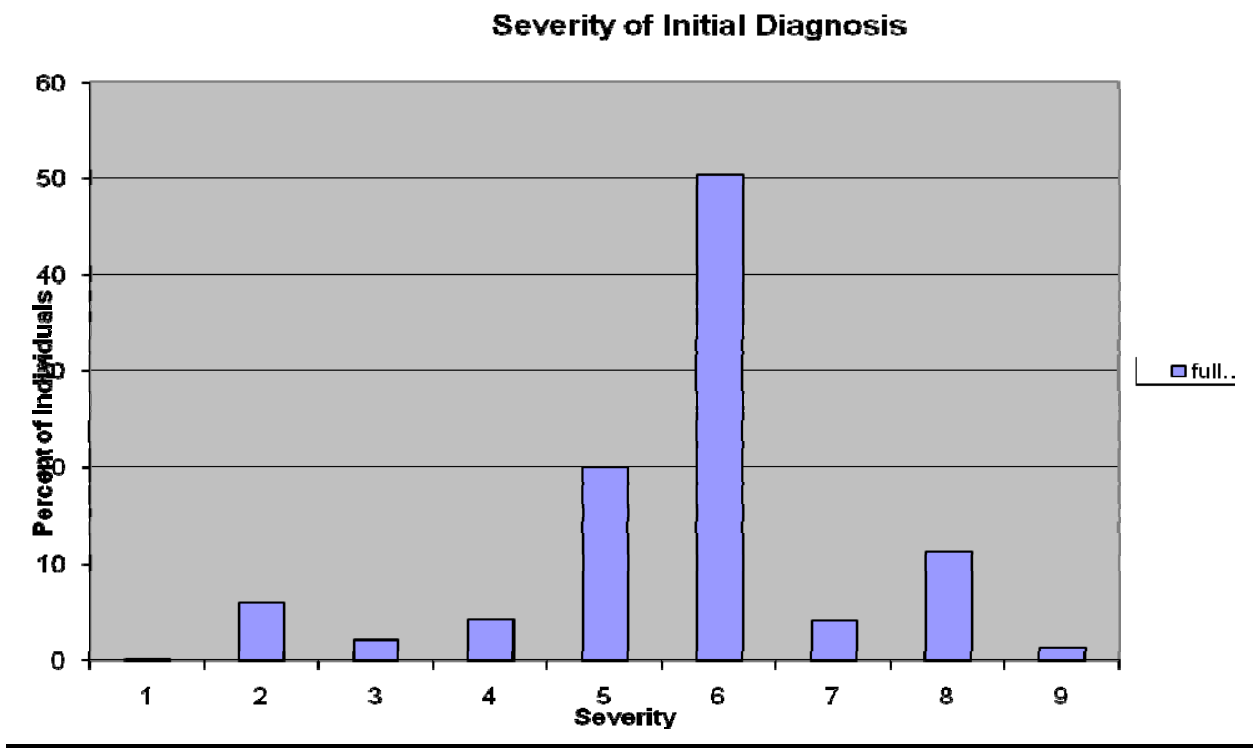

Severity of Initial Diagnosis by Race

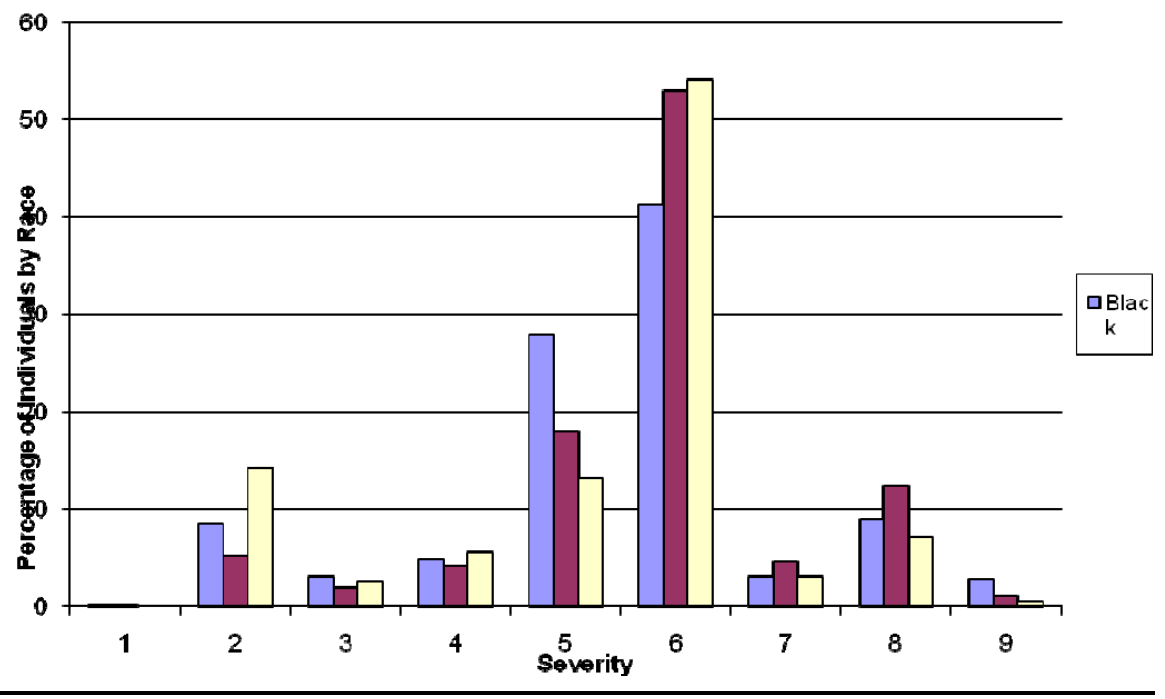




\section{$\underline{\text { References }}$}

1. B.D. Richman, "Insurance Expansions: Do They Hurt Those They Are Designed to Help?” Health Affairs 26, no.5 (2007): 1345-1357.

2. Emmett B. Keeler, et al. "The Demand For Episodes of Medical Treatment in the Health Insurance Experiment” (1988)

3. Joseph Newhouse, et al., "Free For All? Lessons From the RAND Health Insurance Experiment” (1993)

4. M. Gregg Bloche, Race and Discretion in American Medicine, 1 Yale J. Health Pol'y L \& Ethics 95, 108 (2001).

5. Institute of Medicine, Unequal Treatment: Confronting Racial and Ethnic Disparities in Healthcare (2003).

6. United States Surgeon General, Mental Health: Culture, Race, and Ethnicity (Washington: U.S. Government Printing Office, 2001).

7. D.R.Williams and H.W.Neighbors, "Social Perspectives on Mood Disorders," chap. 9 in Textbook of Mood Disorders, ed. D.J. Stein, D.J. Kupfer, and A.F. Schatzberg (Arlington, Va.: American Psychiatric Publishing Inc., 2006), 145-158.

8. D.R.Williams et al., "Prevalence and Distribution of Major Depressive Disorder in African Americans, Caribbean Blacks, and Non-Hispanic Whites,” Archives of General Psychiatry 64, no. 3 (2007): 305-315.

9. Jonathan Gruber, Congressional Testimony, Hearing on Health Benefits in the Tax Code, U.S. Senate Committee on Finance, July 31, 2008.

\footnotetext{
${ }^{1}$ See generally Jonathan Gruber, Health Insurance and the Labor Market 55 (Nat'l Bureau of Econ. Research, Working Paper No. 6762, 1998) (reviewing the empirical literature and concluding that "the results that attempt to control for worker selection, firm selection, or (ideally) both, have produced a fairly uniform result: the costs of health insurance are fully shifted to wages”).

2 B.D. Richman, “Insurance Expansions: Do They Hurt Those They Are Designed to Help?” Health Affairs 26, no.5 (2007): 1345-1357.

${ }^{3}$ See C.C. Havighurst \& B.D. Richman, “Distributive Injustice(s) in American Healthcare,” Law and Contemporary Problems 69, no.4 (2006): 44-45 for a discussion of the limited research on this issue. PPACA's “Cadillac Tax,” scheduled to come into effect in 201x, presumes that insurance premiums amount to equal reductions in take-home pay, and prominent economists-including Jonathan Gruberhave testified that it is as well. Gruber (2008).

${ }^{4}$ UHS has employees living in 97 of North Carolina's 100 counties, but 95\% live in the six counties surrounding the Raleigh-Durham area. The region is home to many urban, suburban, and rural residential areas.
} 
${ }^{5}$ To protect employees' privacy, and to ensure that the data remained deidentified, individual salaries were not released. However, UHS HR categorizes each position by job code, each with a fairly precise salary range and required levels of education, which permitted imputing education and annual income for each individual. Income was determined by the mid-point of the income range for each job code, coded in units of $\$ 10,000$ in 2004 dollars. For job codes where wages are hourly, the hourly rate was multiplied by the individual's full-time equivalent. Job code salary ranges were not available for 2001, so 2001 incomes were imputed for each job code from the salary ranges in 2002-04. Finally, faculty member salaries and the salaries of certain administrators are not determined by job code, thus individuals with these positions are not included in the sample. Anecdotal evidence suggests that results would be even stronger if these high-income individuals remained in the data. Also omitted from the analyses were individuals with missing race data $(\mathrm{N}=784)$.

${ }^{6}$ The dataset is skewed by gender since women are heavily represented in healthcare occupations. Approximately $65 \%$ of the individuals in the dataset are female. Female median income in the sample is nearly identical to median income for males, which is just above the median for males in Durham County.

${ }^{7}$ The BCBS plan imposes $\$ 35$ copayments for unlimited outpatient office visits, whereas the other three plans impose $\$ 35$ copayments for up to twenty in-network visits and a $\$ 100$ deductible plus 50\% coinsurance for all out-of-network visits. The non-BCBS plans also impose some precertification requirements and laboratory and outpatient charges. However important or unimportant these cost-sharing differences are, we control in each analysis for insurance plan, including controlling separately for the three plans, enjoyed by $87 \%$ of the population, that offer identical coverage.

${ }^{8}$ It should be noted that the benefits offices of most large employers should have access to similarly useful data, but very few share their data with researchers. Benefits data of this kind is a valuable resource both to understand healthcare consumption and to explore important health policy questions. We are deeply grateful to UHS Human Resources for its eagerness to explore research questions of both local and national importance. Medicare claims data exhibits some of these advantages, since it follows heterogeneous individuals with known insurance benefits, but it does not cover the working population. 